\title{
Morphofunctional properties of adipose-derived multipotent mesenchymal stromal cells in vitro in ovariectomized mice of different ages
}

\author{
Ustymenko A. ${ }^{1,2}$, Kyryk V. ${ }^{1,2}$, Lutsenko T., ${ }^{1,2}$ Tsupykov O. ${ }^{1,3}$, Butenko G. ${ }^{1,2}$ \\ ${ }^{1}$ State Institute of Genetic and Regenerative Medicine of the National Academy of Medical Sciences of Ukraine, Kyiv, Ukraine \\ ${ }^{2}$ Chebotarev State Institute of Gerontology of the National Academy of Medical Sciences of Ukraine, Kyiv, Ukraine \\ ${ }^{3}$ Bogomoletz Institute of Physiology of the National Academy of Sciences of Ukraine, Kyiv, Ukraine \\ e-mail: alina.n.ustymenko@gmail.com
}

\section{ABSTRACT}

In modern regenerative medicine, adipose-derived multipotent mesenchymal stromal cells (MMSCs) are of particular interest for researchers and clinicians since they are widely used in the treatment of a number of diseases. Given the influence of sex hormones on the function of adipose tissue, a key aspect remains the proper assessment of biological safety and the prediction of the effectiveness of such cell transplants in conditions associated with age-related disorders of ovarian endocrine function.

PURPOSE: to determine the morphofunctional properties of adipose-derived multipotent mesenchymal stromal cells in vitro in young and old ovariectomized mice.

MATERIALS AND METHODS. Disorders of ovarian endocrine function were reproduced on an in vivo model of ovariectomy in young and old female CBA/Ca mice at the age of 2 and 14 months, respectively. ADSCs were obtained from adipose tissue and phenotyped by flow cytometry. The morphological characteristics, proliferative potential, and ability to direct multilineage differentiation in osteogenic and adipogenic directions in vitro were evaluated.

RESULTS. Under the impact of ovariectomy in young animals, there was observed a decrease in the proliferative and clonogenic potential of ADSCs in vitro, which parameters approach the level of old mice. In addition, the osteogenic potential of ADSCs in vitro was reduced, as well as the enhancement of directed adipogenic differentiation was shown while increasing body weight of animals.

CONCLUSION. The results of the study indicate the negative impact of ovariectomy at a young age on the functional activity of ADSCs.

KEY WORDS: ovariectomy; adipose tissue; adipose-derived mesenchymal stromal cells; directed differentiation of stem cell

Today it is important to introduce new approaches to the treatment of the elderly, which are aimed at the use of not only pharmacological drugs, but also advanced technologies of regenerative medicine using cell and tissue therapy. The particular interest of researchers and clinicians is attracted by the application of adipose-derived stem cells (ADSCs) as one of the safest and most accessible sources. Due to the multipotent potential of linear differentiation into specialized cells of different types (adipose tissue, bone, cartilage), they are widely used in plastic and reconstructive surgery of soft tissues and musculoskeletal system, in the treatment of endothelial dysfunction at critical limb ischemia and diabetes, ischemic heart disease, endocrine dysfunction etc.
Since the metabolism of adipose tissue is largely regulated by sex hormones, it is naturally to expect changes in the properties of stem cells derived from this source at menopause. In particular, it has been shown that in postmenopausal women, adipose-derived stem cells have an increased potential for adipogenic differentiation under a short-term replacement therapy of $17 \mathrm{~b}$-estradiol. A common manifestation of menopause is postmenopausal osteoporosis, a disease when osteoclast-mediated bone resorption is ahead osteoblast-mediated bone formation, leading mainly to a trabecular bone loss [1, 2].

Since most of the pathological conditions which require cell therapy using autologous stem cells occur mainly in the elderly, the undeniable 
relevance is to establish the criteria for the biological safety of adipose-derived stem cells obtained during menopause and postmenopausal periods, which are accompanied by estrogen deficiency.

Standard model of estrogen deficiency, typical of menopause in humans, is ovariectomy in laboratory animals. A murine model of ovariectomy has shown that estrogen deficiency influences quantitative and qualitative parameters of stem cell subpopulations in bone marrow. It has been shown that in ovariectomized $(\mathrm{OVx})$ mice, the balance between osteogenic and adipogenic differentiation of bone marrow stem cells is impaired, with the prevalence of adipogenic differentiation $[3,4]$. This also changes the number of progenitor cells of different localization. At present, there is actually no data on the lineage potential of adiposederived stem cells in different directions at estrogen deficiency, which is a significant scientific issue regarding the safety and individual therapeutic efficacy of cell products.

Therefore, the PURPOSE of our study was to establish the characteristics of morphology, proliferative potential and the ability to directed differentiation of ADSCs in vitro in mice with ovariectomy model compared to normal animals of the same age.

\section{MATERIALS AND METHODS}

All animal experiments were carried out in accordance with the international principles of the European Convention for the Protection of Vertebrate Animals Used for Experimental and Other Scientific Purposes (European convention, Strasbourg, 1986) [5], Law of Ukraine "On the Protection of Animals against Cruelty» (No. 3447-IV dated 21/02/2016) [6], as well as all standards of bioethics and biosafety.

Animals. The study used young (2 months) and old (14 months) female $\mathrm{CBA} / \mathrm{Ca}$ mice, which were kept under standard conditions in a vivarium of the Chebotarev State Institute of Gerontology of the National Academy of Medical Sciences of Ukraine under a 12:12 h light/dark cycle with access to water and food ad libitum.

Experimental model of ovariectomy. The animals were anesthetized by intraperitoneal administration of $2.5 \%$ solution of 2,2,2-tribromethanol (Sigma, USA) (400 mg/kg) and we performed bilateral ovariectomy under aseptic condition [7]. The animals of the same age, which had only incisions of the abdominal cavity and isolation of the ovaries without resection (sham-operated), were used as a control. The wounds were sutured in layers and the animals were under the heat source until they recovered from anesthesia. The four experimental groups were formed (Table 1).

In 60 days after the ovariectomy, the animals were euthanized by cervical dislocation under pre-analgesia with $2.5 \%$ solution of 2,2,2-tribromethanol (Sigma, USA) at a dosage $400 \mathrm{mg} / \mathrm{kg}$. Under aseptic condition, subcutaneous adipose tissue was isolated in order to obtain of ADSCs in culture in vitro.

Obtaining of MIMSCs from adipose tissue. Stromal-vascular fraction (SVF) was obtained from the subcutaneous adipose tissue according standard protocol [8]. For this purpose, in sterile conditions, the fragments of adipose tissue were minced with scissors and fermented in $0.1 \% \mathrm{col}$ lagenase type $1 \mathrm{~A}$ solution (Sigma, USA) for 90 min at $37^{\circ} \mathrm{C}$ with constant agitation on a shaker at a rotation speed of $100 \mathrm{rpm}$. The resulting cell suspension was washed in DMEM (Sigma, USA) by centrifugation at $300 \mathrm{xg}$. The supernatant with mature adipocytes was removed and cell pellet passed through a sterile EASYstrainer nylon filter with $100 \mu \mathrm{m}$ pore diameter (Greiner bio-one, Austria). The resulting SVF was plated into $25 \mathrm{~cm}^{2}$ culture flasks for adhesive cells (Nunc, USA) and cultured in complete nutrient medium DMEM-F12 containing $10 \%$ fetal bovine serum (HyClone, USA), $100 \mathrm{U} / \mathrm{ml}$ penicillin, $100 \mu \mathrm{g} / \mathrm{ml}$ streptomycin, (Sigma-Aldrich, USA), 1:100 nonessential amino acids (Sigma-Aldrich, USA), $200 \mathrm{mML}$-glutamine (Sigma-Aldrich, USA) in a $\mathrm{CO}_{2}$ incubator in humidified atmosphere with $5 \% \mathrm{CO}_{2}$ at $+37^{\circ} \mathrm{C}$. The nutrient medium was changed every 3 days. Upon reaching $80 \%$ confluence of the monolayer, the cells were suspended using a mixture of $0.25 \%$ trypsin solution (Sigma-Aldrich, USA) with $0.02 \%$ Versen solution (Bio-Test Laboratory, Ukraine) and subcultured to the $3^{\text {rd }}$ passage. After each passage, the immunophenotype of the cell population was determined.

Immunophenotyping of cells by flow cytometry. The phenotyping of the obtained cells by markers CD44, CD73, CD90, CD45, was performed by flow cytometry using BD FACSAria cell sorter (Becton Dickinson, USA). The samples of $2 \cdot 10^{5}$ cells in $50 \mu$ l CellWash buffer (BD, USA) were incubated with rat anti-mouse monoclonal antibodies conjugated with phycoerythrin (BD Pharmingen, USA) at a working concentration of $0.5 \mu \mathrm{g} / 10^{6}$ cells for $30 \mathrm{~min}$ and washed in CellWash buffer (BD Bioscience, USA) by centrifugation at $300 \mathrm{xg}$ for $5 \mathrm{~min}$. The data were analyzed using $\mathrm{BD}$ FACSDiva 6.2.1 software (Becton Dickinson, USA), collected at least 2•104 cells per sample [9].

Determination of the colony-forming potential of ADSCs. The colony forming potential of ADSCs was evaluated using a colony forming unit assay. The cells of the $2^{\text {nd }}$ passage were plated at a density of 300 cells in $9.5 \mathrm{~cm}^{2}$ Petri dishes in a-MEM culture medium (Sigma, USA) containing $20 \%$ fetal bovine serum (HyClone, USA), 100 units/ml penicillin, $100 \mu \mathrm{g} / \mathrm{ml}$ streptomycin, (Sigma-Aldrich, USA), 1:100 nonessential amino acids (Sigma-Aldrich, USA), $200 \mathrm{mM}$ L-glutamine (Sigma-Aldrich, USA) and cultured in a $\mathrm{CO}_{2}$ incubator in humidified atmosphere with $5 \% \mathrm{CO}_{2}$ at $+37^{\circ} \mathrm{C}$. After 14 days in culture, dishes with adhesive colonies were washed with phosphate-buffered saline (PBS) and stained by Romanovsky-Giemsa. Colonies containing at least 50 cells were counted under a binocular microscope. The plating efficiency (PE) was determined by the formula [10]:

$$
(\mathrm{PE}, \%)=\frac{\text { the number of formed colonies }}{\text { the total number ofplanted cells }} \cdot 100 \%
$$

The proliferative potential of ADSCs. The cells $\left(1 \cdot 10^{5}\right)$ were seeded into a $35 \mathrm{~mm}$ Petri dish and cultured in DMEM-F12 culture medium (Sigma, USA) containing 10\% fetal bovine serum (HyClone, USA), penicillin $100 \mathrm{U} / \mathrm{ml}$, streptomycin $100 \mu \mathrm{g} / \mathrm{ml}$, (Sigma-Aldrich, USA), 1:100 nonessential amino acids (Sigma-Aldrich, USA), 200 mM L-glutamine (SigmaAldrich, USA). After reaching the confluent, the cells of the monolayer were transferred into a suspension using a mixture of $0.25 \%$ trypsin solution and $0.02 \%$ Versen solution (1:3). The resulting cell suspension was centrifuged in a nutrient medium containing $10 \%$ fetal bovine serum (HyClone, USA) at $380 \mathrm{xg}$ for 10 minutes. After counting the number of cells in a Neubauer hemocytometer, $1 \cdot 10^{5}$ cells were seeded into a $9.5 \mathrm{~cm}^{2}$ Petri dish and cultured in the complete nutrient medium. Subculturing was stopped when the number of cells obtained after the last passage was less than $1 \cdot 10^{5}$. The number of passages ranged from 2 to 5 .

The population doubling time (PDT) was calculated for each passage by the formula [11]:

$\mathrm{PDT}=\mathrm{T} / 3,31 \lg \left(\mathrm{Xk}_{\mathrm{N}} \mathrm{X}_{0}\right)$, where:

$\mathrm{T}$ - the time of cell cultivation; $\mathrm{Xk}$ - the number of cells obtained after subculturing; $X_{0}$ - the number of cells at the beginning of the passage.

Directed osteogenic differentiation of ADSCs. For directed osteogenic differentiation, cells were plated into a 24 well plate $5 \cdot 10^{4}$ per well and

\begin{tabular}{|c|c|c|}
\hline \multirow[b]{2}{*}{ EXPERIMENTAL GROUP } & YOUNG & OLD \\
\hline & \multicolumn{2}{|c|}{$\begin{array}{l}\text { THE AGE OF ANIMALS AT THE TIME OF SURGERY } \\
\text { (MONTHS); NUMBER (n) }\end{array}$} \\
\hline Ovariectomy (OVx) & 2 months $(n=17)$ & 14 months $(n=14)$ \\
\hline Control, sham-operated (Ctrl) & 2 months $(n=11)$ & 14 months $(n=12)$ \\
\hline
\end{tabular}


cultured in complete DMEM-F12 culture medium (Sigma, USA) containing $10 \%$ fetal bovine serum (HyClone, USA), penicillin $100 \mathrm{U} / \mathrm{ml}$, streptomycin $100 \mu \mathrm{g} / \mathrm{ml}$, (Sigma-Aldrich, USA), 1:100 nonessential amino acids (Sigma-Aldrich, USA), 2 mM L-glutamine (Sigma-Aldrich, USA). After the culture having reached the subconfluent, the complete culture medium was changed to osteoinductive medium, which consisted of the DMEMF12 culture medium (Sigma, USA) supplemented with $10 \%$ fetal bovine serum, L-ascorbic acid 2-phosphate (0.05 mM), dexamethasone (100 nM) and $\beta$-glycerophosphate $(10 \mathrm{mM})$ [12]. Osteoinductive medium was replaced every three or four days. After 21 days, the monolayers of cells were washed with PBS, fixed with $4 \%$ cooled paraformaldehyde solution for 15 min and stained with Alizarin Red 0 to detect calcium salt deposits in the extracellular matrix. The activity of alkaline phosphatase as a marker of functional activity of osteoblasts was determined by BCIP/NBT (Sigma, USA) staining on monolayers fixed with $4 \%$ paraformaldehyde solution. After 30 min of incubation in dark, the cultures were washed with distilled water and the intensity of staining was evaluated by Kaplow [13]. Semiquantitative analysis of calcium salt deposits was performed by Gregory [14]. The color intensity was evaluated by measuring the optical density on a microplate reader Multiskan EX (Thermo Scientific, USA) at a wavelength of $405 \mathrm{~nm}$.

Directed adipogenic differentiation of ADSCs. For directed adipogenic differentiation, $5 \cdot 10^{4}$ cells per well were plated in a 24-well plate and cultured in complete nutrient medium DMEM-HG (Sigma, USA) containing $10 \%$ fetal bovine serum (HyClone, USA), penicillin $100 \mathrm{U} / \mathrm{ml}$, streptomycin $100 \mu \mathrm{g} / \mathrm{ml}$, (Sigma-Aldrich, USA), 1:100 nonessential amino acids (Sigma-Aldrich, USA), $200 \mathrm{mM}$ L-glutamine. Upon reaching the culture of the subconfluent, the complete culture medium was changed to adipogenic medium, which consisted of DMEM-HG (Sigma, USA) with $10 \%$ fetal bovine serum, dexamethasone $(1 \mu \mathrm{M})$, indomethacin $(200 \mu \mathrm{M})$, isobutylmethylxanthine $(500 \mu \mathrm{M})$ and insulin $(5 \mu \mathrm{g} / \mathrm{ml})$. The adipogenic medium was replaced every 3 days. After 14 days of culturing, the cells were washed with PBS, fixed with $4 \%$ cooled paraformaldehyde solution, and stained with Oil Red S solution (Sigma, USA) to visualize lipid granules in the cytoplasm of cells [15]. After visualizing the granules, were added $100 \%$ isopropanol to each well and incubated for $10 \mathrm{~min}$ at room temperature on a shaker. $250 \mu$ of supernatant were aspirate from each well, and the optical density was analyzed at a wavelength of $500 \mathrm{~nm}$ on a microplate reader Multiskan EX (Thermo Scientific, USA).

Statistical data processing. Statistical data processing was performed by parametric (Student's t-test, Fisher's test) and non-parametric (Wilcoxon-Mann-Whitney U-test) statistics using Statistica 7.0 software (StatSoft Inc., USA). Data are presented as Mean \pm SEM. The statistical difference of indicators between the comparison groups was considered significant at $p<0.05$.
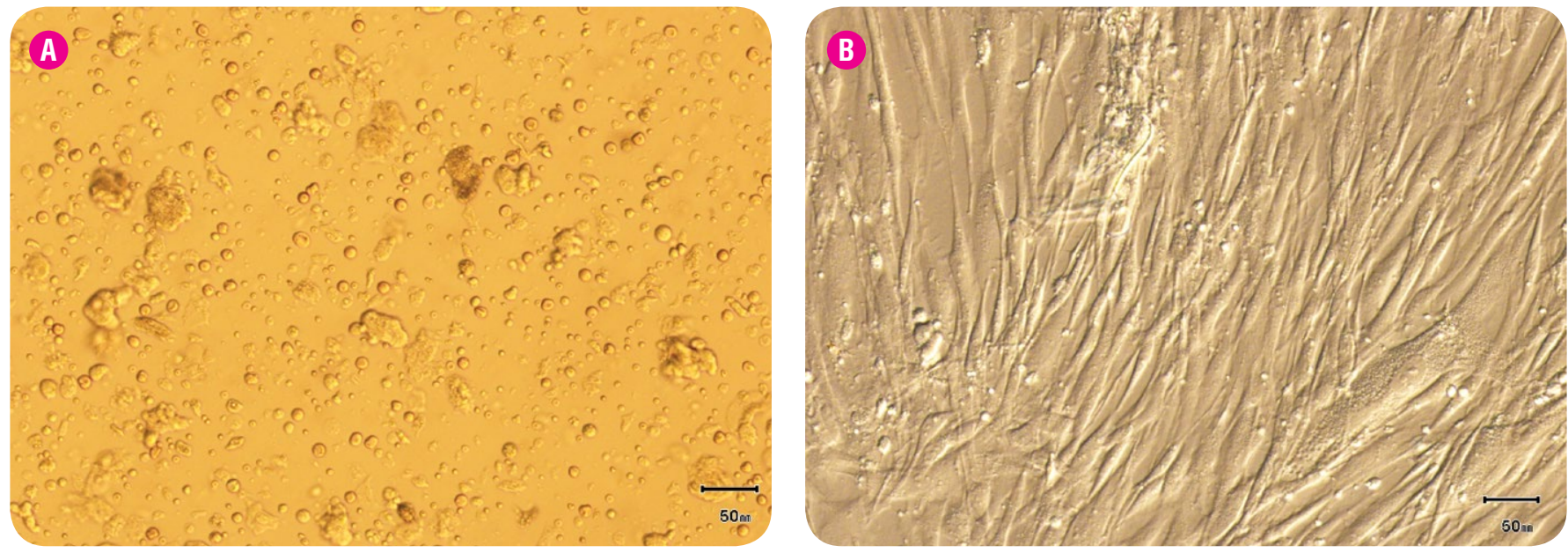

\section{RESULTS AND DISCUSSION}

During the first stage of the study, we obtained primary stromal-vascular fraction of adipose tissue, from which the homogeneous population of stromal cells was obtained during subculturing. It was established that the primary culture of adherent adipose-derived cells of both young and old animals, regardless of ovariectomy, consists of cells different in morphology and size, which is related to the diversity of the initial cell population in SVF (Fig. 1 A).

SVF consists of several cell types, including circulating blood cells, fibroblasts, pericytes and endothelial cells. When replacing the culture medium, the weakly adhesive and non-adhesive cells were eliminated during cultivation. On the $5-7^{\text {th }}$ day of cultivation, the number and size of cells increased, resulting in a large adhesion area and a rate of confluence on the surface of the culture flasks. The diameter of the cells attached to the flask varied from 10 to $250 \mu \mathrm{m}$, with certain cell shapes: fibroblastic, spindle-shaped and stellate, sometimes with multiple processes, with clearly visualized nuclei. On average, after 12-14 days in culture, adhesive cells formed up to $90 \%$ of the confluent monolayer (Fig. 1 B). After subculturing, the cells reached a subconfluent state on average on the $3^{\text {rd }}$ day and consisted mainly of the population of fibroblastic cells.

\section{THE PHENOTYPE OF THE ADSCS CULTURES}

Flow cytometry immunophenotyping of ADSCs cultures of all groups of the first passage revealed the expression of mesenchymal markers CD44 (86.85 $\pm 4.40 \%)$, CD73 (38.9 $\pm 11.9 \%)$ and CD90 (40.85 $\pm 13.7 \%)$, which after subsequent passages increased and corresponded to the phenotype typical for MMSCs (Fig. 2) [16]. Low expression of hematopoietic marker CD45

There were no statistically significant differences in any of the markers between the control groups of both age groups, as well as differences between the groups of animals ovariectomized at young and old age.

\section{COLONY-FORIIING POTENTIAL OF ADSCS}

OF OVARIECTOMIZED MICE ducts quality because it demonstrates both the ability to self-renewal and the ability to differentiate into a specialized adult cell type $[17,18]$.

According to the CFU assay results, a statistically significant difference between the colony-forming ability of ADSCs in young and old mice is shown. Thus, per 300 seeded ADSCs, $31.0 \pm 7.24$ colonies ( $P E=10 \%$ )
CHARACTERISTICS OF ADIPOSE-DERIVED CELL CULTURES

The colony-forming efficacy is an important indicator of cellular pro-

Fig. 1. Microphotograph of the murine primary SVF culture. A - the $1^{\text {st }}$ day of SVF cells culturing. B - the $12^{\text {th }}$ day of SVF cells culturing. Light microscopy. Scale $-50 \mu \mathrm{m}$ 
CD44
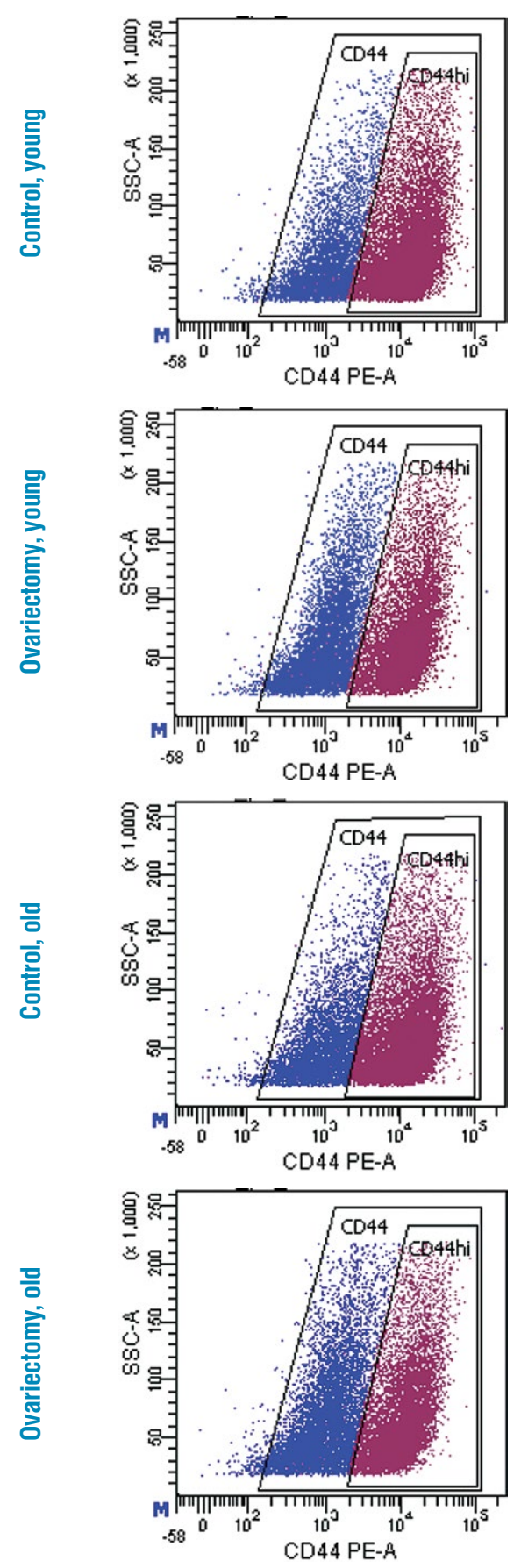

CD73
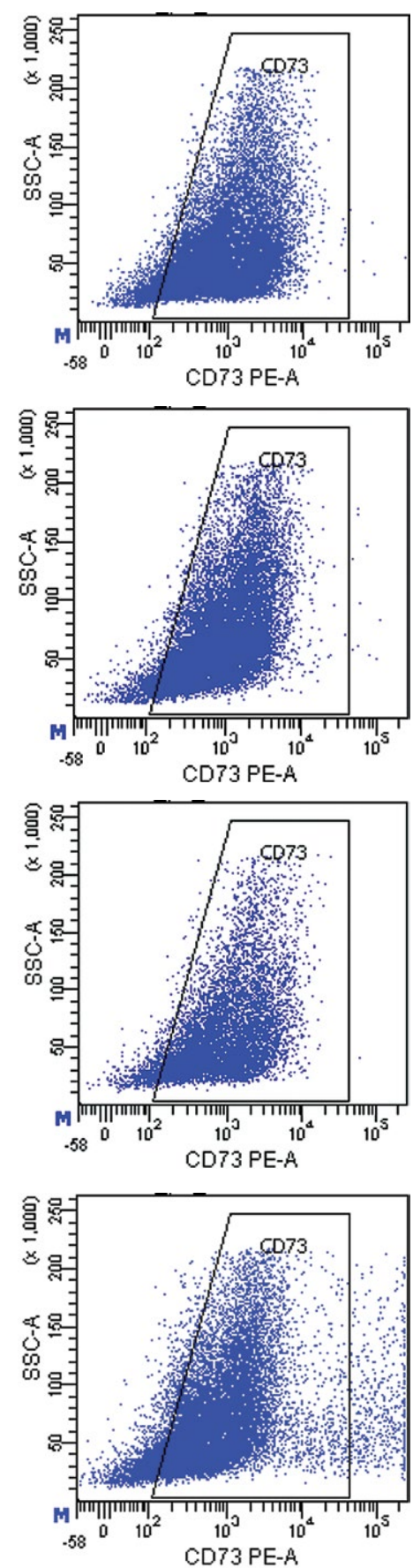

CD90
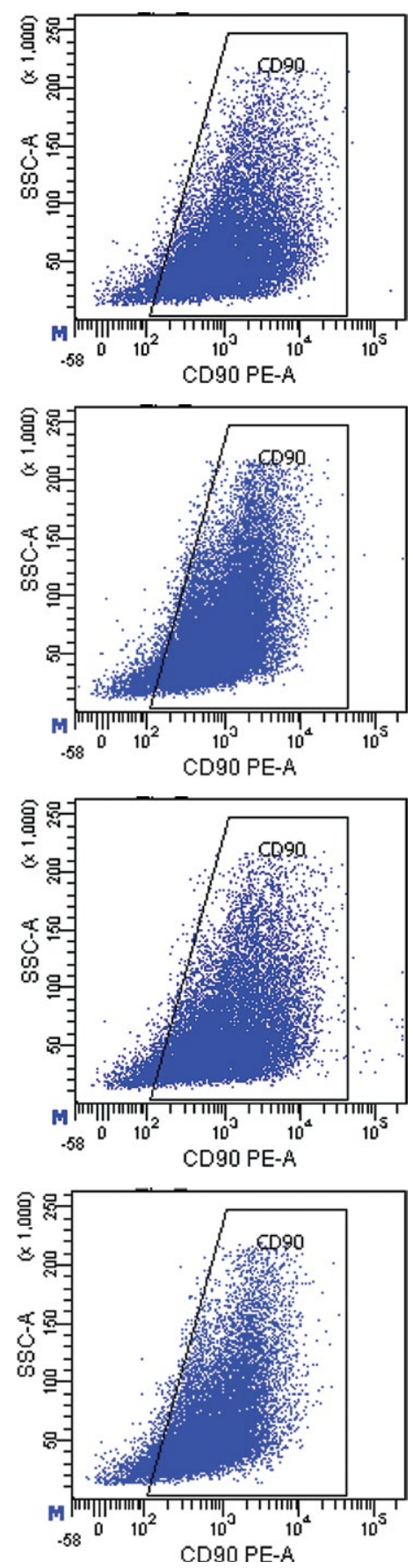

人

Fig. 2. Histograms of stromal markers CD44, CD73 and CD90 expression in the culture of murine ADSCs according to flow cytometry, the $2^{\text {nd }}$ passage.

in the group of young animals with ovariectomy $(n=9)$; and $43 \pm 6.84$ colonies (PE $=14 \%$ ) in the control group of sham-operated animals $(n=6)$ of the same age formed for 14 days $(p<0.05)$. At the same time, no differences were observed between CFU-assay data in the groups of old ovariectomized (31.28 $\pm 12.57 ; \mathrm{n}=7$ ) and sham-operated animals $(30.25 \pm 10.07 ; n=4)$, but significant reduction of the clonogenic potential of ADSCs compared with the control group of young animals $(p<0.05)$ (Fig. 3 ).

\section{THE PROLIFERATIVE POTENTIAL OF ADSCS}

OF OVARIECTOMIZED MICE

Determination of cell growth kinetics in culture in vitro showed that ADSCs of young animals with ovariectomy $(n=7)$ proliferated almost twice as slowly as compared to the control animals $(n=5)$ of the same age $(6.44 \pm 2.26$ and $3.12 \pm 1.02$ days, respectively; $p<0.05)$. At the same time, in old mice with ovariectomy $(n=8)$ and sham-operated ones $(\mathrm{n}=3)$, there was also an increase in population doubling time $(6.82 \pm 2.5$ and $10.86 \pm 4.22$, respectively), and retardation of cell proliferation compared with the young control group (Fig. 4).

\section{THE POTENTIAL OF MURINE ADSCS FOR ADIPOGENIC}

\section{DIFFERENTIATION IN VITRO}

The multipotent properties of murine adipose tissue cultures were confirmed by the ability to directed differentiation in osteogenic and adipogenic directions. 


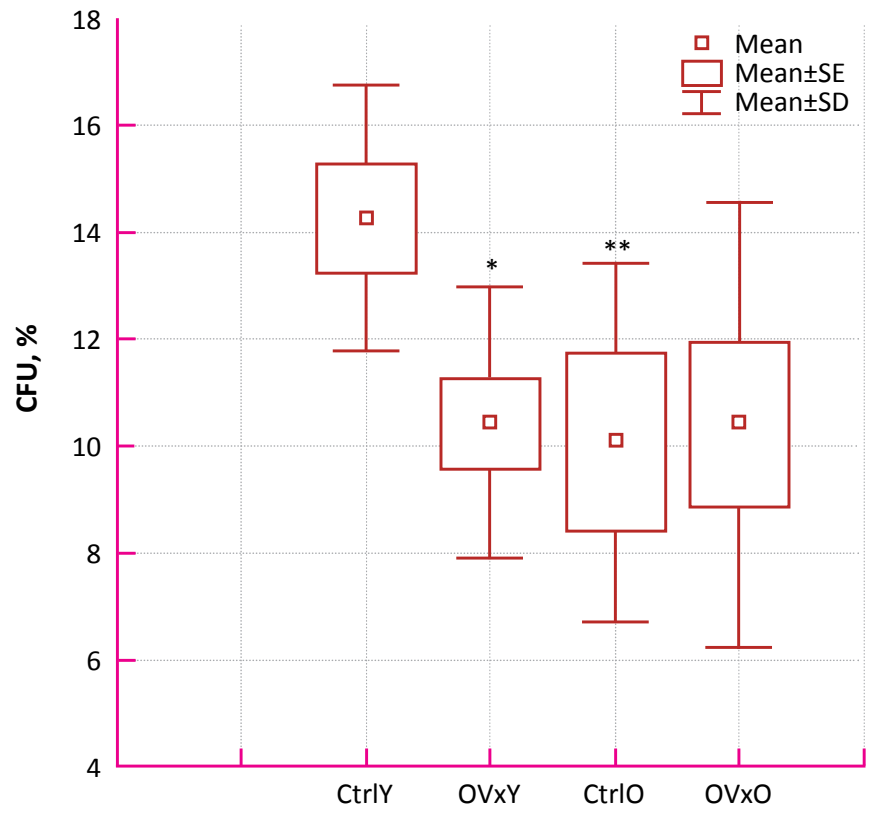

Fig. 3. CFU assay. The efficacy of colony formation of ADSCS in experimental animals. CtrlY - control, young $(n=6)$; OVxY - ovariectomy, young $(n=9)$; CtrlO - control, old $(n=4)$; OVxO - ovariectomy, old $(\mathrm{n}=7)$.

Notes: ${ }^{*}-p<0.05$ compared to the control group of young animals; ${ }^{* *}-p<0.05$ compared to the control group of young animals.

On the $14^{\text {th }}$ day, adipogenic differentiation in vitro occurred in ADSCs cultures of all experimental groups (Fig. 5).

A statistically significant difference between the control and ovariectomized groups of young mice is shown by optical density parameters: ovariectomy contributed to the enhancement of the adipogenic potential in vitro. The degree of differentiation in the groups of old mice was not statistically different from that in the group of young ovariectomized animals (Table 2).

It can be assumed that enhanced adipogenic differentiation of ADSCs in vitro is the cause of the increase in adipogenesis in vivo and subsequent obesity, which is confirmed by a probable increase in the body weight of ovariectomized animals ( $25.04 \pm 1.64 \mathrm{~g} ; \mathrm{n}=17)$ compared to the control group (22.53 $\pm 1.15 \mathrm{~g} ; \mathrm{n}=11)$ of the same age $(\mathrm{p}<0.0001)$ (Fig. 5$)$.

It has been shown that osteogenic differentiation in vitro occurred in ADSCs cultures of all experimental groups. In response to osteogenic induction of ADSCs, on the $21^{\text {st }}$ day of culturing, high alkaline phosphatase activity (Fig. 6) and mineralization of the extracellular matrix by calcium salts were observed (Fig. 7, 8)

Ovariectomy in young animals reduced the potential for osteogenic differentiation manifested in the decrease in the intensity of mineralization of extracellular matrix with calcium salts compared to age-matching control group. There were no statistically significant differences in this parameter

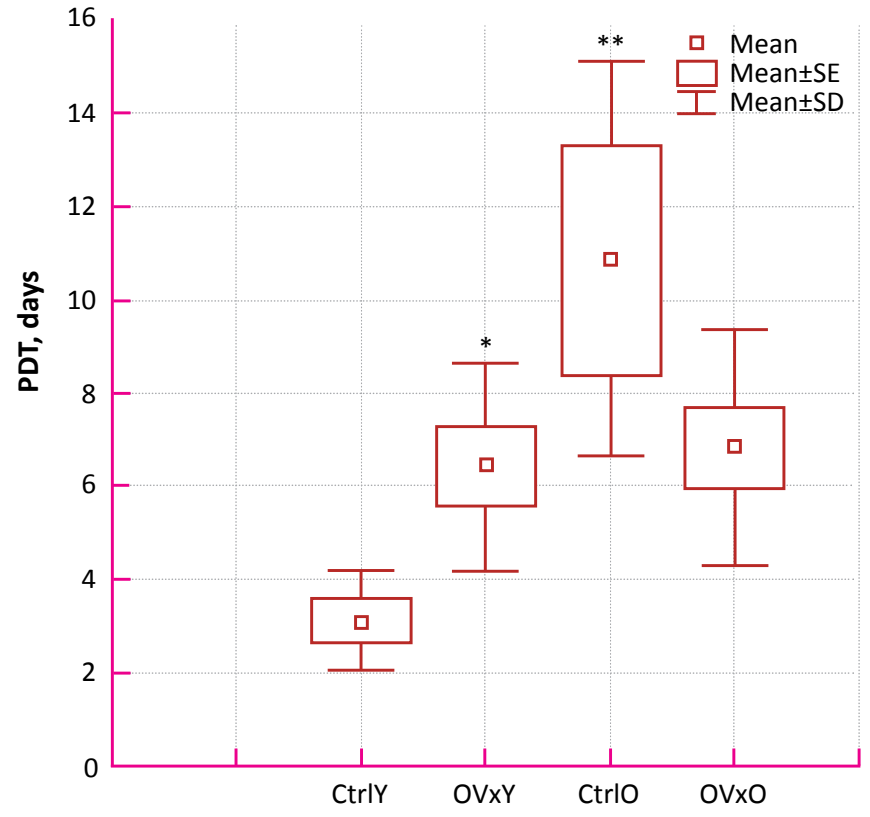

Fig. 4. Results of the PDT test. The number of days required to doubling the population of cells in culture in vitro. CtrlY - control, young $(n=5)$; OVXY - ovariectomy, young $(n=7)$; CtrlO - control, old $(n=3)$; OVxO - ovariectomy, old $(n=8)$.

Notes: ${ }^{*}-p<0.05$ compared to the control group of young animals; ${ }^{* *}-p<0.05$ compared to the control group of young animals.

in the old animals of both groups, but there was a decrease in the optical density compared to the control group of young animals (Table $\mathbf{3}$ ).

Thus, the stromal-vascular fraction was isolated from the adipose tissue of experimental $\mathrm{CBA} / \mathrm{Ca}$ line mice of both age groups with ovariectomy and sham surgery. MMSCs were cultured and proliferated in culture in vitro under standard culture conditions and with the addition of specific factors. It is shown that heterogeneous SVF contains a population of cells characterized by high adhesive properties, a phenotype, characteristic of MMSCs, and the ability to multilineage differentiation in osteogenic and adipogenic directions.

Thus, according to the results of immunophenotyping, the obtained cell populations correspond to the MMSCs immunophenotype due to the high CD73, CD90 and CD44 expression and the low CD45 expression [16]. However, no statistically significant differences were observed for any of the markers between the groups of sham-operated and ovariectomized mice of both age groups, as well as differences between the $\mathrm{OVxY}$ and $\mathrm{OVxO}$ groups [19]. Yet, ovariectomy at a young age leads to a decrease in the proliferative, clonogenic potential of ADSCs to the level of old animals $[20,21]$. In this case, adipogenic differentiation in vitro is increased compared to the control group of the matching age, which may cause an increase in vivo adipogenesis and body weight after ovariectomy in the young $[22,23,24]$.

Table 2. Parameters of optical absorption of the culture medium after staining ADSCS with Oil Red dye at directed adipogenic differentiation, a. u.; light absorption at wavelength of $500 \mathrm{~nm}$.

YOUNG

OLD

\begin{tabular}{|c|c|c|c|c|c|c|c|}
\hline \multicolumn{2}{|c|}{ CTRL } & \multicolumn{2}{c}{ OVx } & \multicolumn{2}{c}{ CTRL } & OVx \\
\hline CM & AIM & CM & AIM & CM & AIM & CM & AIM \\
\hline $\begin{array}{c}0.096 \pm 0.10 \\
(n=6)\end{array}$ & $\begin{array}{c}0.148 \pm 0.10^{*} \\
(n=6)\end{array}$ & $\begin{array}{c}0.128 \pm 0.12 \\
(n=14)\end{array}$ & $\begin{array}{c}0.287 \pm 0.10^{* \#} \\
(n=8)\end{array}$ & $\begin{array}{c}0.132 \pm 0.04 \\
(n=8)\end{array}$ & $\begin{array}{c}0.232 \pm 0.10^{*} \\
(n=8)\end{array}$ & $\begin{array}{c}0.114 \pm 0.01 \\
(n=8)\end{array}$ & $\begin{array}{c}0.183 \pm 0.01 * \\
(n=12)\end{array}$ \\
\hline
\end{tabular}

Notes: CM - a standard complete medium; AIM - a growth medium with adipogenic differentiation factors; * $-p<0.05$ compared to control cultures (without ADF) of animals of the same age; \# - $p<0.05$ compared to young animals of same age and culture conditions. 
Standard culture medium
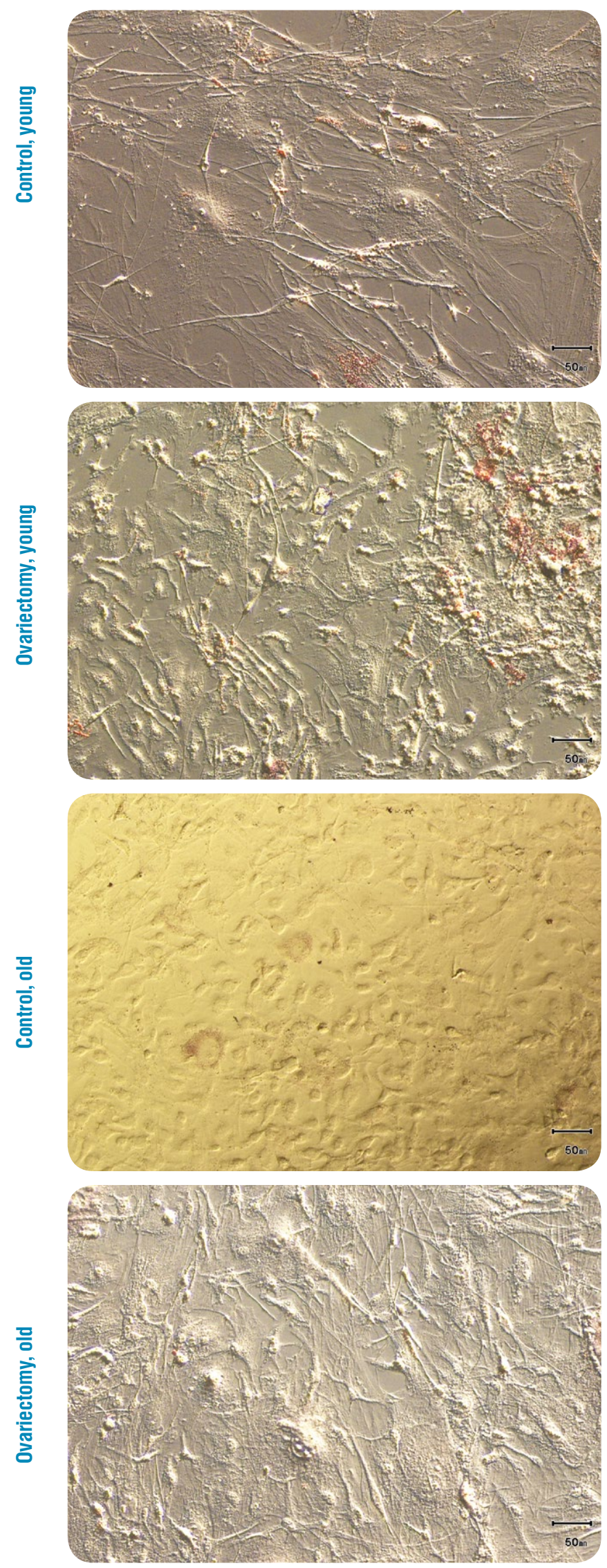

Adipogenic induction medium
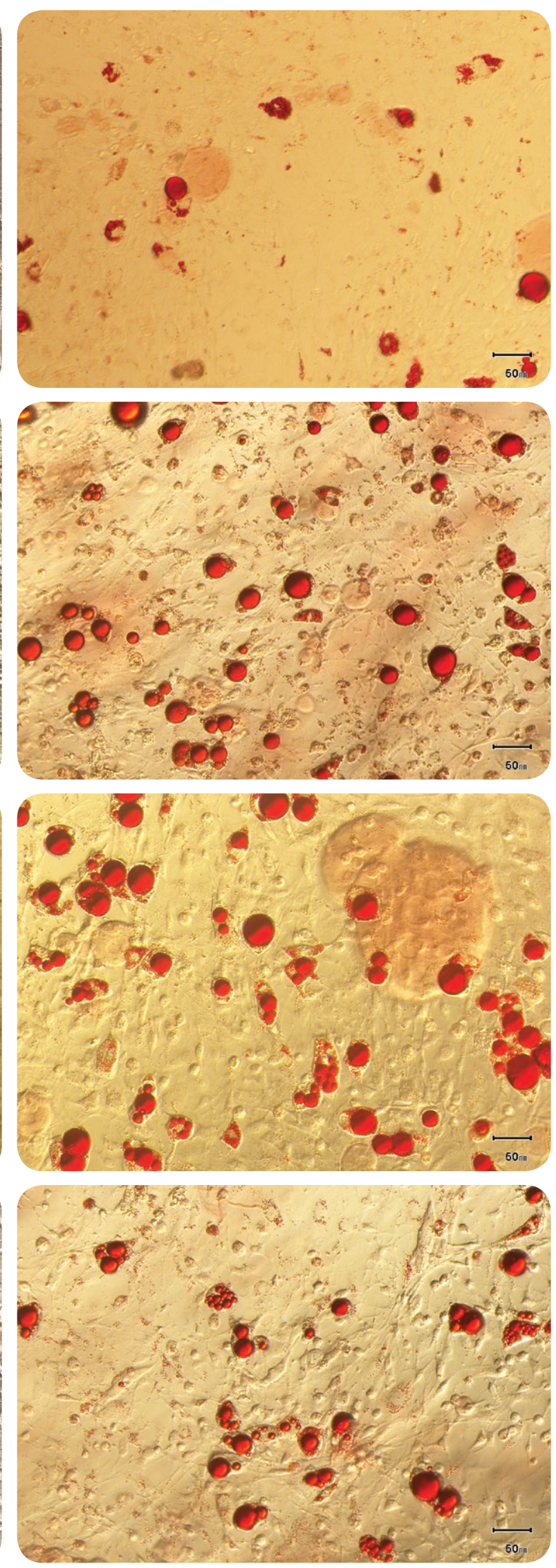

A Fig. 5. Microphotographs of murine ADSCs cultures after directed adipogenic differentiation. Cells with lipid inclusions are stained with Oil Red (red). Light microscopy, scale $-50 \mu \mathrm{m}$. 
Table 3. Parameters of optical absorption of the culture medium after staining ADSCs with Alizarin Red S dye at osteogenic differentiation, a. u.; light absorption at wavelength of $405 \mathrm{~nm}$.

\begin{tabular}{|c|c|c|c|c|c|c|c|}
\hline \multicolumn{4}{|c|}{ YOUNG } & \multicolumn{4}{|c|}{ OLD } \\
\hline \multicolumn{2}{|c|}{ CTRL } & \multicolumn{2}{|c|}{ OVx } & \multicolumn{2}{|c|}{ CTRL } & \multicolumn{2}{|c|}{ OVx } \\
\hline $\mathrm{CM}$ & OIM & $\mathrm{CM}$ & OIM & $\mathrm{CM}$ & OIM & $\mathrm{CM}$ & OIM \\
\hline $\begin{array}{c}0.35 \pm 0.10 \\
(n=4)\end{array}$ & $\begin{array}{c}0.59 \pm 0.14^{*} \\
(n=4)\end{array}$ & $\begin{array}{c}0.29 \pm 0.06 \\
(n=8)\end{array}$ & $\begin{array}{c}0.37 \pm 0.09^{\star \#} \\
(n=8)\end{array}$ & $\begin{array}{c}0.26 \pm 0.07 \\
(n=7)\end{array}$ & $\begin{array}{c}0.38 \pm 0.07^{* a} \\
(n=7)\end{array}$ & $\begin{array}{c}0.28 \pm 0.09 \\
(n=8)\end{array}$ & $\begin{array}{c}0.39 \pm 0.09 * a \\
(n=8)\end{array}$ \\
\hline
\end{tabular}

Notes: CM - a standard complete medium; OIM - a growth medium with osteogenic differentiation factors; ${ }^{*}-p<0.05$ compared to cultures cultured without osteogenic growth factors; $\#-p<0.05$ compared to the control group of the same age and culture conditions; $a-p<0.05$ compared to the control group of young animals and culture conditions.

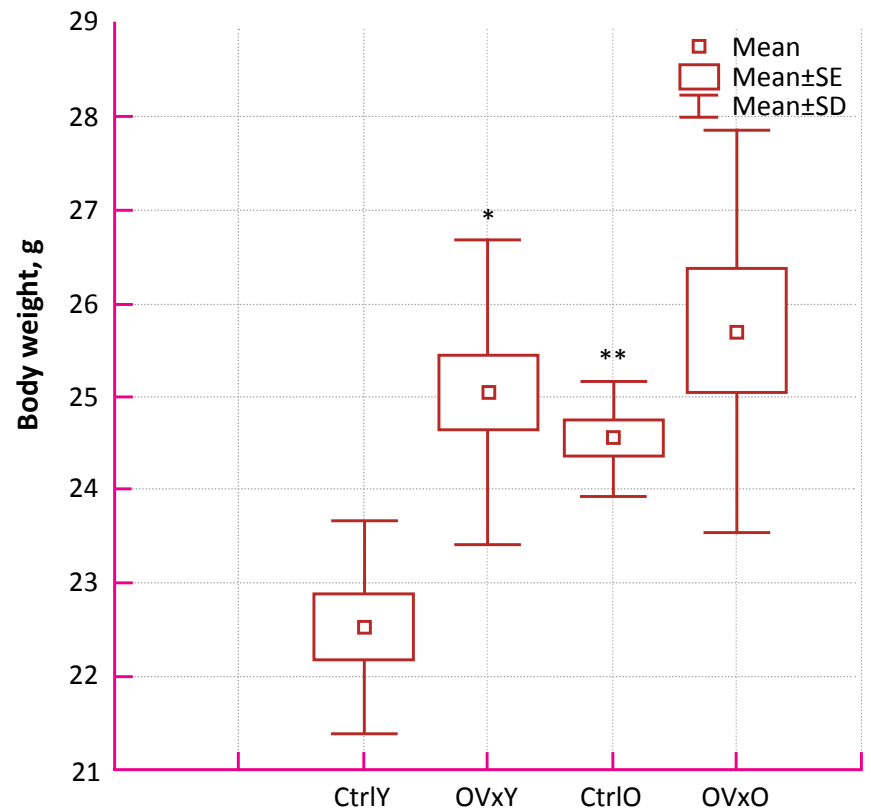

Fig. 6. The body weight of experimental animals, g. CtrlY - control, young $(n=11)$; OVXY - ovariectomy, young $(n=17)$; CtrlO - control, old $(\mathrm{n}=12) ; \mathrm{OV} \times 0$ - ovariectomy, old $(\mathrm{n}=11)$.

Notes: ${ }^{*}-p<0.0001$ compared to age-matching control; ${ }^{* *}-p<0.002$ compared to young control animals.

We have shown that ovariectomy leads to a decrease in osteogenic potential of ADSCs in CBA/Ca mice, ovariectomized at a young age compared to ADSCs of control animals of the matching age, which completely confirms the leading role of estrogens in osteogenic differentiation of MMSCs, in particular the role of $17 \beta$-estradiol in supporting growth and differentiation through the ERa receptor [25]. In addition, 17 $\beta$-estradiol stimulates MMSCs for the expression of osteogenic genes of alkaline phosphatase, collagen type I and TGF- $\beta 1[26,27]$.

Thus, the results of the study suggest that ovariectomy and associated estrogen deficiency affect the functional characteristics of ADSCs in vitro in young mice, and during aging certain mechanisms of the reduction of the clonogenic and proliferative potentials of stem cells are activated, which makes them less dependent on the ovaries. Understanding the effects of sex hormones on stem cells will allow researchers and clinicians to modulate the function of these cells in vitro in order to obtain the maximum therapeutic effect for the treatment of various diseases and avoid side effects.

\section{CONCLUSION}

1. In young CBA/Ca mice after ovariectomy, the population doubling time of ADSCs in vitro is increased compared to the sham-operated animals of the same age.

2. Ovariectomy of CBA/Ca mice at a young age is likely to increase lipid accumulation in cell vacuoles at adipogenic differentiation in vitro compared to control group of animals of the same age.

3. More pronounced potential for adipogenic differentiation of ADSCs in vitro in young ovariectomized animals may indicate to increased adipogenesis in vivo and induction of subsequent obesity, as evidenced by the probable increase in body weight in young ovariectomized animals compared to the control group of the same age. 
Standard culture medium
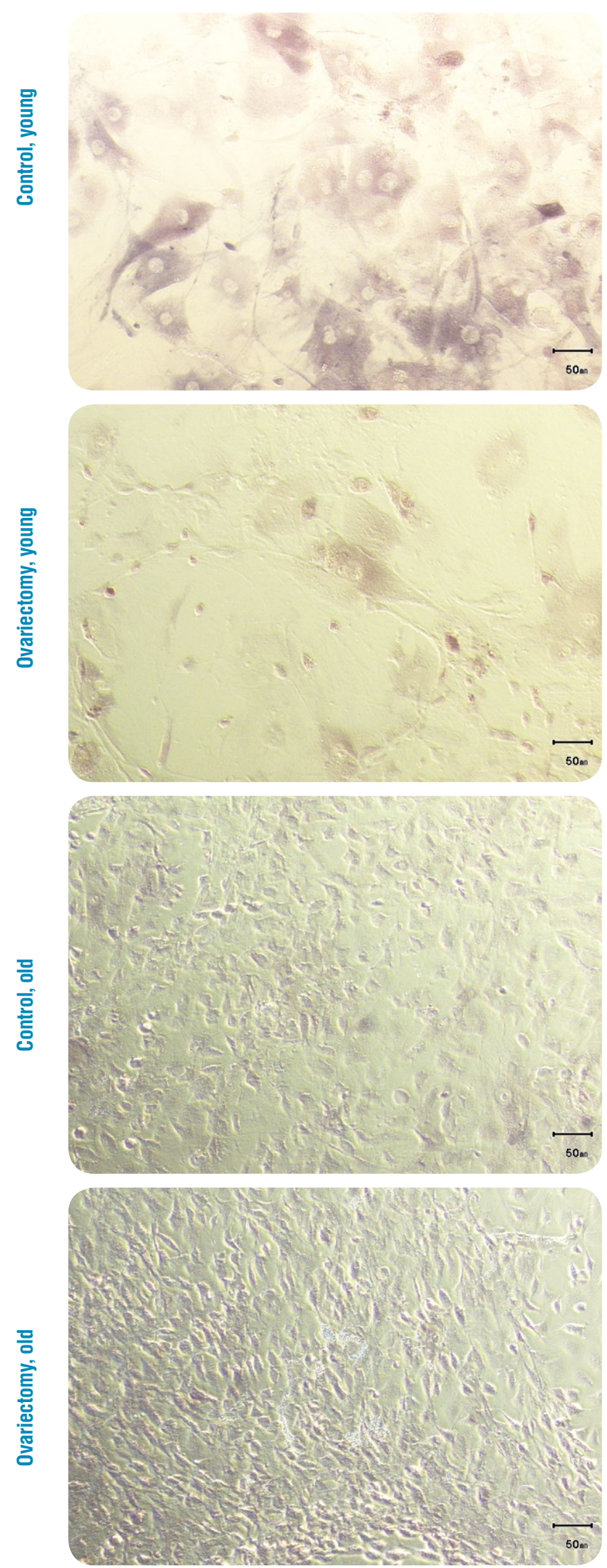

Osteogenic induction medium
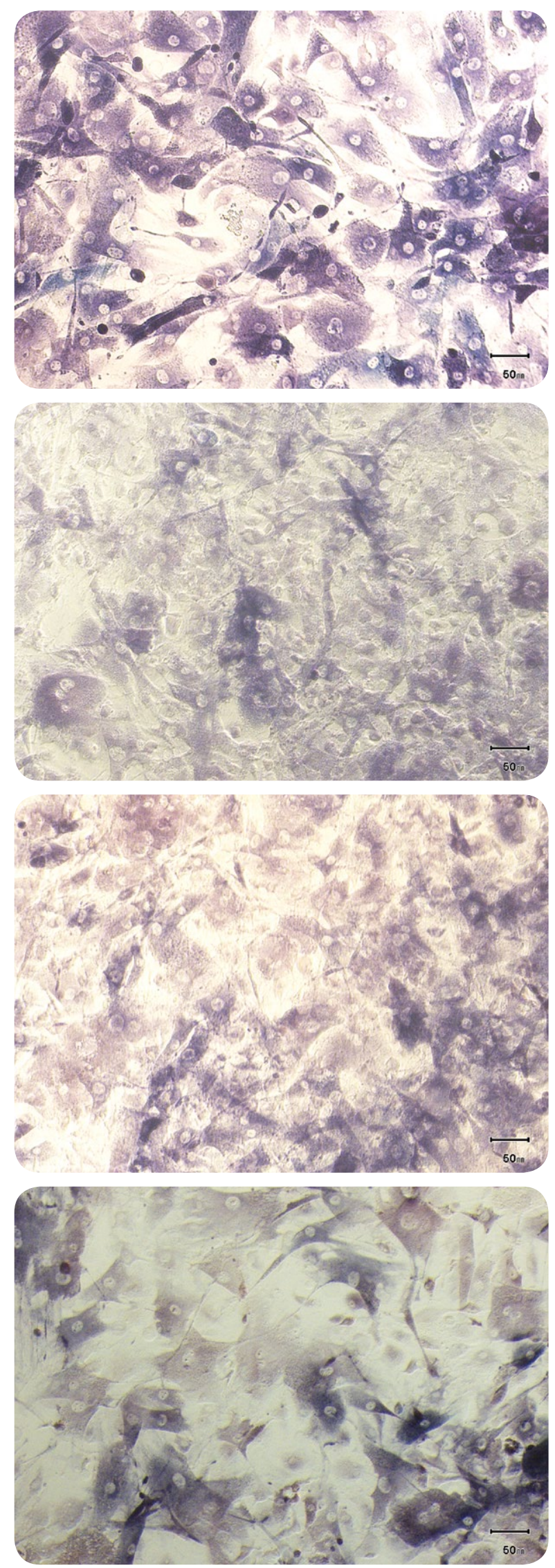

A Fig. 7. Microphotographs of ADSCs cultures on the $2^{1 \text { st }}$ day of culturing in growth medium without differentiation factors (control) and with osteogenic differentiation factors. Cytochemical staining of alkaline phosphatase using BCIP-NBT dye. Light microscopy, scale $-50 \mu \mathrm{m}$. 
Standard culture medium
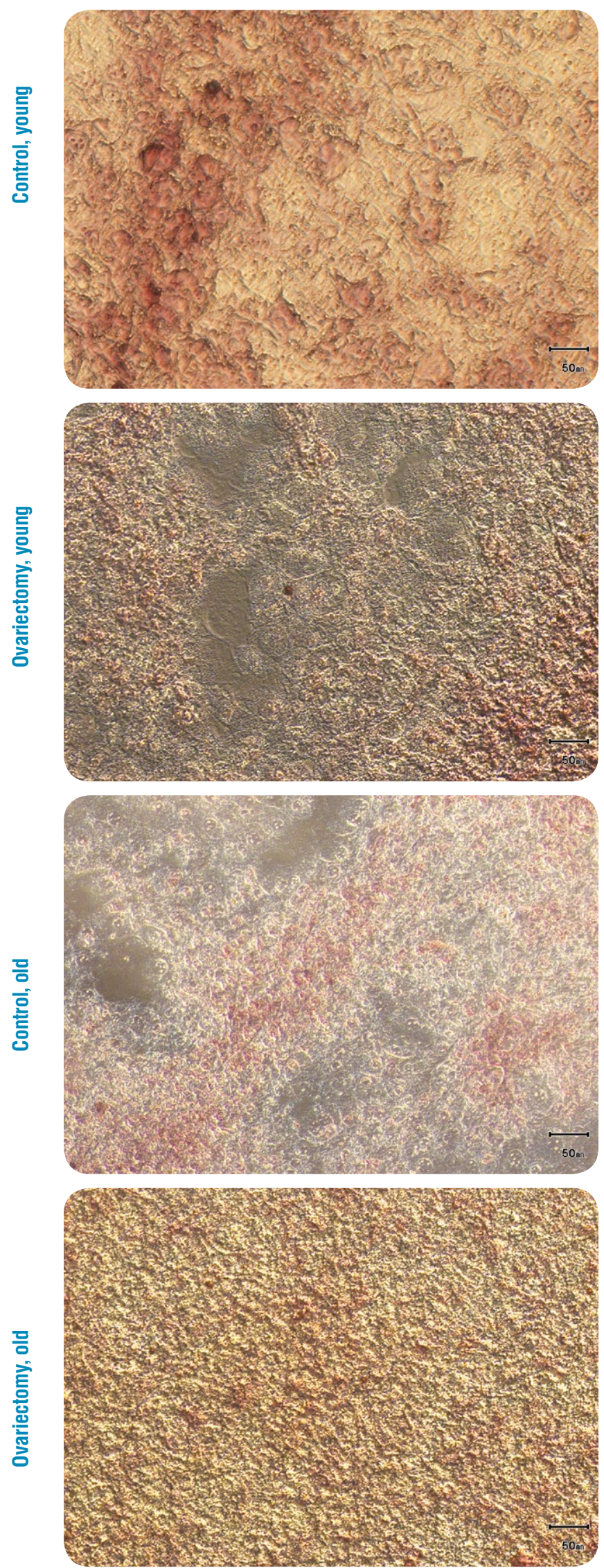

Osteogenic induction medium
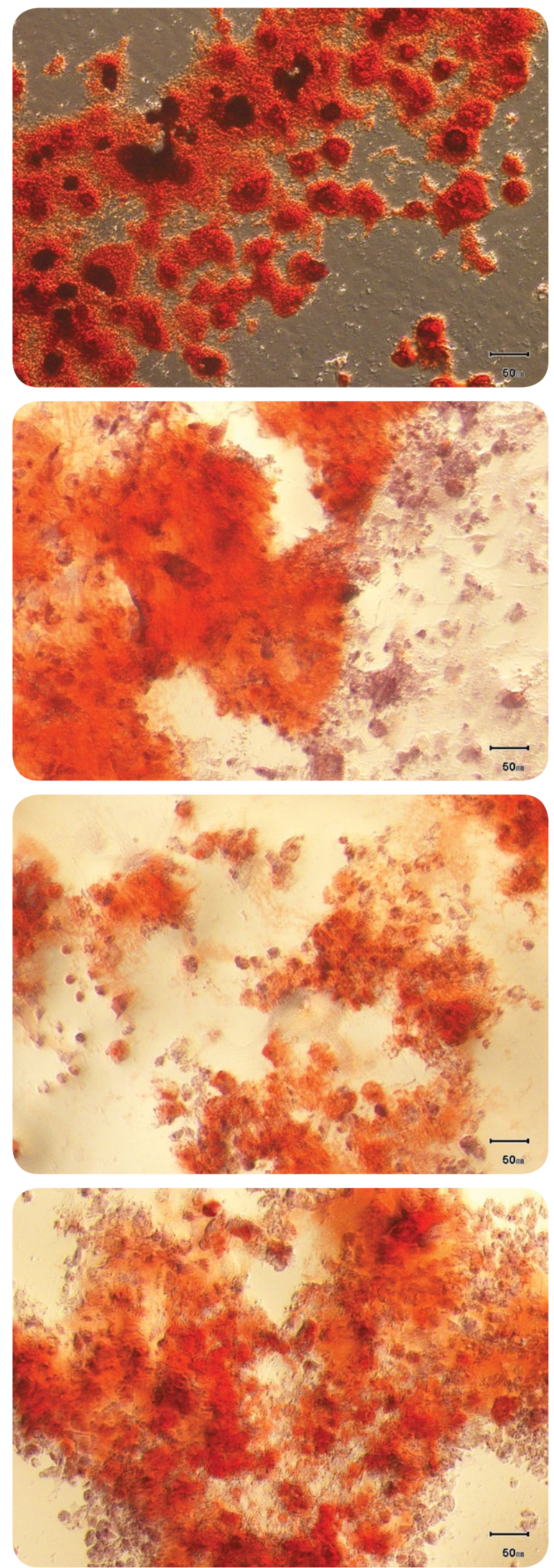

A Fig. 8. Microphotographs of ADSCS cultures on the $21^{\text {st }}$ day of culturing in growth medium without differentiation factors (control) and with osteogenic differentiation factors. Staining of extracellular matrix mineralization with calcium salts by Alizarin Red S dye. Light microscopy, scale $-50 \mu \mathrm{m}$ 


\section{REFERENCES}

1. Eastell R, O'Neill TW, Hofbauer LC, LangdahI B, Reid IR, Gold DT, et al. Postmenopausal osteoporosis. Nat Rev Dis Primers. 2016; 2:16069. DOl: 10.1038/nrdp.2016.69.

2. Clowes JA, Riggs BL, Khosla S. The role of the immune system in the pathophysiology of osteoporosis.Immunol Rev. 2005; 208:207-227.

3. Nuttall ME, Gimble JM. Controlling the balance between osteoblastogenesis and adipogenesis and the consequent therapeutic implications. Curr Opin Pharmacol. 2004; 4:290-294.

4. Rodriguez JP, Astudillo P, Rios S, Pino AM. Involvement of adipogenic potential of human bone marrow mesenchymal stem cells (MSCs) in osteoporosis. Curr Stem Cell Res. 2008; 3:208-218.

5. European Convention for the Protection of Vertebrate Animals used for Experimental and Other Scientific Purposes. Strasbourg, 18.III.1986. https://rm.coe. int/168007a67b.

6. https://zakon.rada.gov.ua/laws/show/3447-15.

7. Standard operating procedure \#206. Rodent ovariectomy. McGill. https://www.mcgill.ca/research/files/research/206_-_rodent_ovariectomy.pdf.

8. Gang Yu, Xiying Wu, Gail Kilroy, et al. Isolation of Murine Adipose-Derived Stem Cells. Adipose-Derived Stem Cells: Methods and Protocols, Methods in Molecular Biology. DOl 10.1007/978-1-61737-960-4_3.

9. Maecker HT, Trotter J. Flow cytometry controls, instrument setup, and the determination of positivity. Cytometry A. 2006; 69(9):1037-42.

10. Plating Efficiency. Adapted from Mather J. P., and Roberts P. E., 1998. Introduction to Cell and Tissue Culture: Theory and Technique. Plenum Press. New York and London. https://fscimage.fishersci.com/webimages_FSC/downloads/HyClone_Protocol_5.pdf.

11. Freshney RI. Culture of Animal Cells, $5^{\text {th }}$ ed. New York: Wiley-Liss; 2005.

12. Li J, Mareddy S, Tan DM, Crawford R, et al. A minimal common osteochondrocytic differentiation medium for the osteogenic and chondrogenic differentiation of bone marrow stromal cells in the construction of osteochondral graft.Tissue Engineering. 2009; 15(9):2481-2490.

13. Kaplow L. A Histochemical Procedure for Localizing and Evaluating Leukocyte Alkaline Phosphatase Activity in Smears of Blood and Marrow. Blood. 1955; 10:10231029.

14. Gregory $C A$, Gunn WG, Peister $A$, et al. An alizarin red-based assay of mineralisation by adherent cells in culture: comparision with cetylpyrydinum chloride extraction. Anal Biochem. 2004; 329: 77-84.

15. Ruiz-Ojeda F, Rupérez A, Gomez-Llorente C, et al. Protocols for Adipogenic Differentiation Assays for Characterization of ASC. Cell Models and Their Application for Studying Adipogenic Differentiation in Relation to Obesity: A Review. Int J Mol Sci. 2016; 17(7):1040. DOl: 10.3390/ijms17071040.

16. Dominici M, Le Blanc K, Mueller I, et al. Minimal criteria for defining multipotent mesenchymal stromal cells. The International Society for Cellular Therapy position statement. Cytotherapy. 2006; 8(4):315-17.

17. Friedenstein AJ, Chailakhyan RK, Latsinik NV, Panasyuk AF, Keiliss-Borok IV. Stromal cells responsible for transferring the microenvironment of the hemopoietic tissues. Cloning in vitro and retransplantation in vivo Transplantation. 1974; 17:331-340.

18. Prockop DJ. Marrow stromal cells as stem cells for nonhematopoietic tissues. Science. 1997; 276: 71-74.

19. Pachon-Pena G, Yu G, Tucker A, Wu X, Vendrell J, Bunnell BA, et al. Stromal stem cells from adipose tissue and bone marrow of age-matched female donors display distinct immunophenotypic profiles. J Cell Physiol. 2010; 226:843-851. DOl: 10.1002/jcp.22408.

20. Meichen Liu, Hua Lei, Ping Dong, et al. Adipose-Derived Mesenchymal Stem Cells from the Elderly Exhibit Decreased Migration and Differentiation Abilities with Senescent Properties. Cell Transplantation. 2017; 26(9):1505-1519.

21. Chen $\mathrm{HT}$, Lee MJ, Chen $\mathrm{CH}$, et al. Proliferation and differentiation potential of human adipose-derived mesenchymal stem cells isolated from elderly patients with osteoporotic fractures. J Cell Mol Med. 2012; 16:582-592. DOI: 10.1111/j.1582-4934.2011.01335.x.

22. Fu Y, Li R, Zhong J, Fu N, Wei X, et al. Adipogenic differentiation potential of adipose-derived mesenchymal stem cells from ovariectomized mice. Cell Prolif. 2014; 47:604-614. DOl: 10.1111/cpr.12131.

23. Justesen J, Stenderup K, Ebbesen E, Mosekilde L, Steiniche T, Kassem M. Adipocyte tissue volume in bone marrow is increased with aging and in patients with osteoporosis. Biogerontology. 2001; 2:165-171.

24. Hong L, Colpan A, Peptan IA. Modulations of 17-beta estradiol on osteogenic and adipogenic differentiations of human mesenchymal stem cells. Tissue Eng. 2006; 12:2747-53.

25. Wang Q., et al. Temporal expression of estrogen receptor alpha in rat bone marrow mesenchymal stem cells. Biochem Biophy Res Commun. 2006; 347:117-23.

26. Zhou $S$, et al. Estrogen modulates estrogen receptor alpha and beta expression, osteogenic activity, and apoptosis in mesenchymal stem cells (MSCs) of osteoporotic mice. J Cell Biochem. 2001; 36:144-55.

27. Leskela H.V., et al. Estrogen receptor alpha genotype confers interindividual variability of response to estrogen and testosterone in mesenchymal-stem-cell-derived osteoblasts. Bone. 2006; 39:1026-34.

\begin{tabular}{|c|c|}
\hline TRTICL ON THE SITE \\
\hline TRANSPLANTOLOG.ORG \\
\hline
\end{tabular}

The authors declared no potential conflicts of interest with respect to the research, authorship, and/or publication of this article.

Received: September 23, 2019

Accepted: November 30, 2019 